\title{
RESEARCH ON QUALITY SUPERVISION AND SPOT CHECKS TECHNOLOGY FOR NATIONWIDE LARGE-SCALE TOPOGRAPHIC MAPS IN CHINA
}

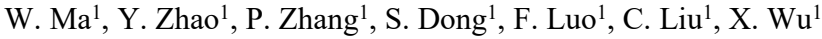 \\ ${ }^{1}$ National Quality Inspection and Testing Center for Surveying and Mapping Products, Beijing 100830, China - (maweichn, \\ 2310931713, 819376859, 610482824, 1254020931, 188926006, 84924075)@qq.com
}

Commission I, WG I/10

KEY WORDS: Large-scale Topographic Maps, Quality Supervision and Spot Checks, Sampling, Prior Knowledge

\begin{abstract}
:
Large-scale Topographic Maps (LST-Maps) play an important role in China, so their quality is of great importance. In order to guarantee the quality of the survey and mapping achievements, China has been implementing a supervision and inspection system. There are relatively big differences in the content, mode of production and demands for application of the LST-Maps all over the country, making it difficult for current informatization level of supervision and traditional methods of quality control to become fair and efficient. The existing operational mode, sampling means, and technical standards of inspection applied in supervision are not entirely suitable for the needs in quality supervision and inspection of LST-Maps. In face of the abovementioned contradictions and problems, the research has combined the requirements of quality supervision and spot checks of all levels. Various achievements such as an index system, technical means, inspection procedures and a software platform have been created, and they are widely used in the quality supervision and spot checks of national and provincial LST-Maps over the past three years.
\end{abstract}

\section{INTRODUCTION}

Large-scale Topographic Maps (LST-Maps, scale from 1:500 to $1: 10000)$ provide important basic data for territorial spatial planning, management of natural resources, investigation and designing, and engineering construction in China. It has a direct impact upon the quality of construction and the decisionmaking of the government over scientific matters. It is not only a realistic need to ensure and continuously improve the quality of the LST-Maps, so as to safeguard national sovereignty and the people's interests, but it's also a mandatory requirement for the sustainable development in surveying and mapping, as well as in geographic information industry. With the overall requirement of strengthening the operational and postoperational supervision, and in order to guarantee the quality of the survey and mapping achievements, China has been implementing a supervision and inspection system. Within legal provisions, the competent departments of surveying at all levels conduct quality supervision and spot checks over the quality of LST-Maps.

Considering the strict requirements of quality supervision and spot checks, namely legality, fairness, openness, a high frequency of supervision and a high quantity of tasks, LSTMaps are characterized by their high accuracy in position, a detailed expression of terrain and their features. There are relatively big differences in the content, mode of production and demands for application of the LST-Maps all over the country, making it difficult for the informatization of supervision and traditional methods of quality control to become fair and efficient. The existing operational mode, sampling means, and technical standards of inspection applied in supervision are not entirely suitable for the needs in quality supervision and inspection of LST-Maps. In face of the abovementioned contradictions and problems, and under the requirements of the random inspections by randomly selected officers/experts and requiring the prompt release of results (also called "Double Random, One Open" policy), the project has combined the requirements of quality supervision and spot checks of all levels. Researches and development of quality supervision and spot checks technology are carried out for the surveying and mapping results of LST-Maps. Various achievements such as an index system, technical means, inspection procedures and a software platform have been created, and they are widely used in the quality supervision and spot checks of national and provincial LST-Maps over the past three years.

\section{PROBLEMS AND POSSIBLE SOLUTIONS}

Problem 1: With a large number of projects, various types of scales, a scattered regional distribution, different background of inspection experts, there is a high subjectivity in the procedure of manual sampling, it is thus difficult to guarantee the practice of "Double Random, One Public" principle during quality supervision and spot checks.

Possible solutions 1: with the preconditions of the regulations of supervision and spot checks being strictly followed, we may research and develop quantitative-random sampling technology for supervision and spot checks; develop a delaminating random sampling technology based on our prior knowledge; ensure the legality, openness and transparency of the projects and inspection experts when samplings are taken, and a scientific randomness in the sampling of results.

Problem 2: Since there are significant differences in the content, mode of production (Zebker et al, 1994) and requirements of application of LST-Maps throughout the country, together with the characteristics of topographic maps, such as a high accuracy in their position and a detailed expression of terrain and its features, the existing quality inspection and evaluation standards are insufficient. They are not suitable to solve the issues we face with the quality inspection and assessment of the LST-maps throughout the country. 
Possible solutions 2: we should fully refer to the existing standards of production and quality inspection, take the requirements of supervision and spot checks into consideration, as well as take into account the regional differences of the nationwide LST-Maps, conduct researches and design an evaluation index system for the quality inspection of LSTMaps. By doing the above, we will be able to achieve the scientificalness, reasonableness, universality and manipulability of the index system; we will also be able to unify the technical caliber in this aspect throughout the whole country.

Problem 3: In view of the high frequency of supervision and spot checks, a high number of tasks, a narrow time frame and uneven technical capabilities of inspection experts, the technical methods in the existing standards are relatively backward and the efficiency is relatively low. They are not fully applicable for the quality inspection or assessment of LST-Maps throughout the country.

Possible solutions 3: we can make full use of the advantages of the current advanced equipment for quality inspection, then research and develop the "internal and field work integration" method for the quality inspection and assessment of LST-Maps based on the quality inspection supporting database. This can ensure the consistency of the use of technical methods and evaluation standards nationwide.

Problem 4: There are strict regulations and complicated procedures for supervision and spot checks, as well as numerous factors affecting the fairness of inspection. The low extent of informatization in traditional procedures of supervision and spot checks also makes it difficult to meet the requirements of fairness and efficiency in the inspection process.

Possible solutions 4: research and development of a collaborative and efficient information platform for quality inspection for LST-Maps, combined with sampling technologies, the quality inspection and evaluation index system and the quality inspection and evaluation technical methods, which can improve the automation level of key processes in quality inspection.
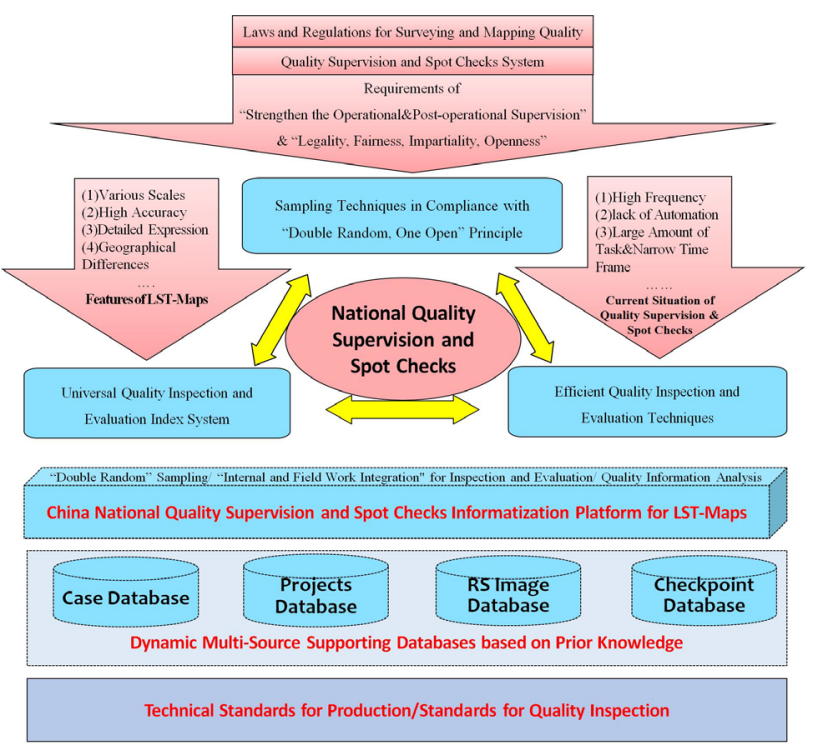

Figure 1. Problems and Overall Concept of Solutions

\section{MAJOR TECHNOLOGICAL INNOVATIONS}

According to the abovementioned problems and solutions, we conducted research by surveying, researching, experimenting and software developing. We have completed the research of sampling technologies, an index system and technical methods of inspection, and we have built an inspection software platform.

\subsection{Sampling techniques have been improved}

3.1.1 Sampling techniques for projects and experts in quality supervision and spot checks have been developed: Based on the delaminating ideas, the "project data pool" and "expert data pool" related with nationwide LST-Maps were established, which enables the realization of summary, statistics and analysis of projects and expert information. According to the principle of quality supervision and spot checks, and the "Double Random" inspection principles, a quantitative-random sampling technique was designed and implemented. There is now a full coverage of supervision and spot checks of 31 provincial administrative region (currently excluding Hong Kong, Macao and Taiwan). There is also a coverage of projects of various scales, and the types of the sampled projects. On the basis of randomness of supervision and spot checks, we have focused on key projects involving national sovereignty and security, projects involving personal and property safety, major national or collective interests and social impact.

There is a random selection of quality inspection experts who have considered their professional expertise and the avoidance mechanism, ensuring the openness and transparency of the projects for spot checks. The whole procedure of "Double Random" spot checks is recorded, and the relevant personnel is held accountable for any possible problems.

3.1.2 A delaminating random sampling technique based on prior knowledge was developed: In view of the large number of topographic maps, differences in the qualities, a wide distribution of results, and obvious differences in terrain, combined with the actual situations and requirements of internal and external inspections, the idea of "sampling lots for supervision and spot check" was proposed based on the existing sampling methods of quality inspection. This concept considers the characteristics of spot checks and various results (i.e. prior knowledge including the type of results, types of difficulties, distribution of results, topographical categories, traffic conditions, etc.), suitable for different delaminating solutions and sampling techniques of various sampling units. Not only can this satisfy the requirements of supervision and spot checks, but this can also make the samples more representative and minimize errors. Furthermore, it can prevent the sampling from becoming too focused or missing certain quality characteristics. Such can in turn avoid the manual sampling becoming too subjective, which would hinder us to reach the goal of supervision and spot checks.

\subsection{An inspection index system was researched and designed}

3.2.1 Established a classification index system for the inspection: Developed a templated, dynamic configuration technology for indicators: According to the current national standards for production, focusing on the specificities of supervision and spot checks, a quality model (Ma et al, 2018) for LST-Maps has been researched and designed. It has created a content classification index system consisting of the 
characteristics of quality, inspection content and inspection indicators. The design also included individual modules of quality features such as mathematical foundation, positional accuracy, accuracy of attributes, integrity, logical consistency, time accuracy, image/grid quality, characterization quality, data and structural correctness, geographic accuracy, decoration quality and annex quality. It has fully considered the actual situation of production design and focused on different types of results. Inspection indicators were designed for the inspection of results, solutions were created for a comprehensive selection of results and template management.

3.2.2 The research established a quality evaluation index system, and designed the weight adjustment methods of the quality characteristics: The development focuses on the standards of quality inspection of the current surveying and mapping of results, designs and constructs a national quality evaluation index system for the surveying and mapping of the results of LST-Maps. It has also standardized the procedures to measure the quality of results, after considering a reasonable verification method and an evaluation method based on prior knowledge. It has combined the advantages of the current standards of quality inspection for the surveying and mapping of results, in order to create a method to adjust the quality of LST-Maps. It has laid a technical foundation for the evaluation of automation, significantly improved the scientific nature of the evaluation, and achieved a dynamic configuration and scientific combination of the classification index system. A quality assessment index system of the supervision content was implemented.

\subsection{Inspection techniques have been innovated}

3.3.1 An inspection method for LST-Maps has been researched: as specific attributes of LST-Maps constraint with each other, based on their constraints or consistencies, an analytic method of defining the standardization of constraints, also to analyze, calculate and judge has been researched and implemented. It has overcome several difficulties. For example, the traditional SQL statements could only interpret the attributes of topographic maps, perform simple checks on constraint relationships. It was challenging to express the constraint relationships in a unified and standardized way, or to undergo more complicated checks on the constraint relationships. It is now possible to automate the checks of the constraint relationships of the attributes.

\subsubsection{Developed a technical method called "internal and} field work integration" for quality inspection and evaluation of LST-Maps: In view of the high frequency of supervision and spot checks, a high number of tasks, a narrow time frame, uneven capabilities of inspectors, we have tried to make full use of the current advanced inspection equipment to develop technical methods for the mapping results of LST-Maps, based on the "internal and field work integration" principle. This has guaranteed the consistency of technical methods and evaluation scales across the whole country, and it's a breakthrough from the existing standards, the technical methods which are still relatively backward, the low efficiency which are fully adapted to the LST-Maps across the country. They are not entirely suitable for quality inspection and assessment of results in supervision and spot checks either.

\subsection{Constructed a software platform}

In view of the strict requirements of the procedures of supervision and spot checks, the high number of inspection procedures, and the factors affecting the fairness of the inspection, we've fully investigated the requirements for an automated check of the mapping results. We've also combined the sampling techniques, the quality inspection and evaluation techniques, the design of quality inspection and technical methods for evaluation, before developing a informatized platform for the supervision and spot checks of LST-Maps which are collaborative and highly efficient. This includes a web-based projects submitting system, quantitative-random sampling tools for projects, prior-knowledge based sampling tools for LST-Maps, templated configuration tools for inspection contents and indicators, some entry inspection tools based on logical constraints, a cross-platform field inspection and testing tools for mathematical precision test, spatialization marking tools for quality defects based on the symbol library, units' quality scoring tools for LST-Maps, generate and output tools for the inspection records and reports, tools for quality information management and dynamic analysis. All of the above have greatly improved and guaranteed the automation of the key nodes of quality inspection, and have also ensured the legality and fairness of all the inspection activities.

\section{CONCLUSION}

\subsection{Promotion and Application Status}

The technical methods of quality inspection developed by this research have been effectively applied to the national-level supervision and spot checks in 2016, as well as those at the provincial-level over the past 3 years. Among them, the national-level supervision and spot checks cover 462 Grade-A surveying and mapping qualification units and 2052 projects, which are under the jurisdiction of 31 provincial surveying and mapping authorities. As a result, there were zero objection and appeals from the inspection units, which are widely recognized by the competent surveying and mapping authorities.

The sampling and quality inspection platform developed in this project have been promoted nationwide. It has effectively standardized the provincial supervision and spot checks; in view of the nationwide supervision and spot checks, the project has also improved its efficiency and accuracy.

In accordance with the principle of "supervision in accordance with regulations, fairness, high efficiency, openness and transparency", the research has made innovations in the national supervision and management mode for the surveying and mapping. It has also established a multi-element quality supervision and spot check mechanism under "national and provincial linkage", which has further optimized the workflow of quality supervision. Moreover, the research has unified the evaluation scales and standards, ensured the fairness, impartiality and openness of the procedures of supervision and spot checks, thus effectively improved the government's credibility.

The promotion and application of this research has comprehensively and accurately grasped the real quality information of the nationwide LST-Maps, strengthened the operational and post-operational supervision, enhanced the quality awareness among the surveying and mapping qualification units, effectively promoted the implementation of the tenure system to ensure quality, also continuously promoted the quality of LST-Maps. These have provided the necessary support of quality information for the industry regulation conducting by the surveying and mapping authorities, promoted the overall services of the surveying and mapping, served the 
society, served the purposes of improving the livelihood, and provided an accurate, reliable surveying and mapping information for national economic and social development.

It has ensured the seriousness and legality of throughout all the activities of spot checks, also ensured their openness and fairness, the accuracy of the spot check results, the high efficiency of quality inspection. The promotion and application of this research has also provided an accurate and detailed support of quality data for the competent department of surveying and mapping at all levels, fully grasping the overall quality of the industry. This will enable a continuous improvement of the quality of surveying and mapping results throughout the country, achieving remarkable economic and social benefits.

\subsection{Analysis of effectiveness}

The quantitative-random sampling techniques developed by the research guarantees legitimacy and disclosure of the supervision and spot checks, achieving a 100\% transparency of the sampling process. Based on prior knowledge, this sampling technology can reduce sampling risks by approximately $90 \%$, hence meets the needs of "Double Random" principles of the sampling procedures.

The quality inspection of LST-Maps and the evaluation index system developed in the research have been adopted by the provincial surveying and mapping authorities, achieving the basic unification of the technical caliber across the country.

In the research, the techniques methods of "internal and field work integration" for quality evaluation have been adopted by the provincial surveying and mapping authorities. This has helped to achieve the uniformity of the technical methods for quality inspection and evaluation scales of LST-Maps across the country. The efficiency has increased by more than $20 \%$.

The research has designed and continuously updated a dynamic, multi-source supporting database for quality inspection, which can shorten the internal working period by about $10 \%$ and the field inspection workload by more than $30 \%$. At the same time, it also provides a more accurate and detailed quality information for surveying and mapping authorities at all levels, and has more power in supporting the government's quality supervision.

\section{ACKNOWLEDGEMENTS}

This work is partly supported by National Science Foundation of China (grant No.41671440).

\section{REFERENCES}

Zebker, H. A., Werner, C. L., Rosen, P. A., \& Hensley, S., 1994. Accuracy of topographic maps derived from ERS-1 interferometric radar. IEEE Transactions on Geoscience and Remote Sensing, 32(4), 823-836.

Ma, W., Zhang, J., Zhao, Y., Zhang, P., Dang, Y., \& Zhao, T., 2018. Design and Establishment of Quality Model of Fundamental Geographic Information Database. ISPRSInternational Archives of the Photogrammetry, Remote Sensing and Spatial Information Sciences, 1249-1252. 